\title{
How noninvasive investigation has modified our therapeutic approach in vascular medicine
}

\section{PL Antignani}

Italian Society for Vascular Investigation

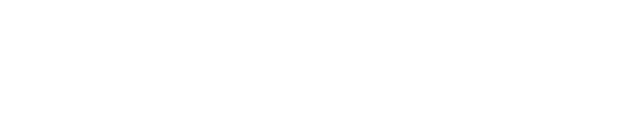

Noninvasive diagnostic methods have modified our therapeutic decision-making in several vascular diseases. In particular, many forms of surgical treatment, both endovascular and open, are performed based exclusively on evaluation with duplex scanning. The purpose of noninvasive ultrasound testing is to distinguish normal from pathological vessels, to classify a wide range of disease states, to assess the collateral circulation, and to do so in a safe and cost-effective manner. The primary aim is to identify patients who are at risk for acute and chronic vascular disease and who may require specific treatment. A secondary aim is to document progressive or recurrent disease in patients already known to be at risk. Of course, individual vascular laboratories must validate their own results against a suitable gold standard, and they have to guarantee the best quality and maximum accuracy. ${ }^{1}$

With regard to diseases of the carotid artery, color flow duplex scanning is the investigation of choice for diagnosis and measurement of carotid stenosis, provided that objective criteria are used and scanning is done by experienced operators. Several velocity criteria used to detect the presence and severity of carotid artery disease and the morphological evaluation of lesions allow us to have a specificity of $90 \%$ and a sensitivity of $99 \%$ when all categories of carotid disease are considered. On the basis of these criteria, we can identify the best therapeutic approach for specific pathological conditions.

Concerning plaque characteristics, surface ulceration, low gray-scale median $(<25)$, heterogeneous appearance of the plaque, and juxtaluminal location of the echolucent area after image normalization are ultrasonographic indicators of plaque vulnerability, and should be considered in selection of appropriate therapy and frequency of follow-up after surgical treatment, ie, carotid endarterectomy or carotid artery stenting. Transcranial color Doppler can be used before carotid endarterectomy or carotid artery stenting to evaluate coexisting lesions of the intracranial vessels, efficiency in the circle of Willis, the intracranial hemodynamic effects of extracranial carotid lesions, cerebrovascular reserve, microembolic events due to ulcerated plaques, cross-clamping risk, and indication for shunting.

Duplex ultrasonography is the ideal method of determining the adequacy of renal artery revascularization, and is helpful in detecting important areas of restenosis after endovascular therapy, ie, percutaneous angioplasty with stent deployment. The renal duplex examination includes spectral Doppler velocities for the renal arteries, renal parenchyma, and abdominal aorta. Peak systolic and end-diastolic velocities obtained
Correspondence: PL Antignani

President of Italian Society

for Vascular Investigation,

Vascular Center Nuova Villa Claudia,

Via Germanico 21 I, 00192 Rome, Italy

Email antignani@mclink.it
Journal of Vascular Diagnostics 2013:I I-3

(C) 2013 Antignani, publisher and licensee Dove Medical Press Ltd. This is an Open Access article

Dovepress

http://dx.doi.org// 0.2147/JVD.S44754 
in branches of the renal artery at the level of the medulla are used to calculate the renal resistive index, a value reflecting the health of the renal parenchyma itself. In addition, the examination should define the pole-to-pole length of each kidney.

Concerning abdominal aortic aneurysm in asymptomatic patients, ultrasound detects the presence of an abdominal aortic aneurysm accurately and at low cost, with a sensitivity and specificity approaching $100 \%$. Ultrasound is ideal for screening and determination of aneurysmal growth rate. A growth rate $>0.7 \mathrm{~cm}$ in six months or $1 \mathrm{~cm}$ in a year has been suggested as a threshold for proceeding to surgery, irrespective of size.

Acute conditions, such as wall dissection, wall rupture, rapid growth, and acute thrombosis, can be identified accurately on ultrasound. Aneurysms repaired by endografts and endovascular stents have unique ultrasound characteristics. Position and patency of the endograft, diameters and pulsatility of the aneurysm, endoleak (sensitivity 81\%-100\%, specificity 74\%-99\%), patency of other vessels, infections, and fistulas are conditions that can be evaluated in the follow-up of endovascular treatments.

The purpose of noninvasive testing for peripheral arterial disease is to confirm a clinical diagnosis and define further the level and extent of obstruction. A variety of algorithms can be used to diagnose peripheral arterial disease noninvasively in the vascular laboratory. These include the ankle brachial index, segmental limb pressures, and exercise treadmill testing. Standardized criteria relating waveform changes to anatomical site and hemodynamic severity of disease are used in diagnostic interpretation. The goal of examination with duplex ultrasound is to elucidate the location and severity of arterial stenosis in the extremities, and can be helpful particularly when planning therapy for known peripheral arterial occlusive disease.

Compared with the gold standard of arteriography, duplex Doppler evaluation used to detect significant stenosis in patients with proximal lower extremity arterial disease demonstrates a high sensitivity $(82 \%)$ and specificity $(92 \%)$. Use of color and pulsed wave Doppler increases the sensitivity $(87 \%-88 \%)$ and specificity $(95 \%-99 \%)$ in identification of stenosis.

For obstruction, accuracy reaches 100\%. Standard surveillance protocols recommend ultrasound evaluation of the graft twice during the first postoperative year, and annually thereafter. Early intervention improves long-term patency by $15 \%-20 \%$. Technical failure often causes graft failure in the first month. Intima hyperplasia and progression of atherosclerotic disease can cause graft failure over the ensuing years. All these conditions can be evaluated accurately using duplex ultrasound.

Colour flow duplex scanning can provide both morphological and hemodynamic information and now represents a rapid alternative noninvasive method of diagnosing deep vein thrombosis in the lower limbs. At present, this method is considered the "goal standard" and reaches an accuracy of $100 \%$ if we use the compression ultrasound maneuver. Colour flow duplex scanning represents a valid clinical tool, not only for initial diagnosis of deep vein thrombosis but also for assessment of the long-term outcome of thrombus. This test can guide initial patient management, providing information about clot attachment at the vein wall and resolution. In addition, it can identify patients potentially at high risk for post-thrombotic syndrome. Finally, Duplex ultrasound may be used to compare and evaluate the results of different anticoagulant and fibrinolytic drug regimens with regard to the long-term outcome of venous thrombus in a lower extremity.

In the evaluation of chronic venous disease, preoperative evaluation (venous mapping) is best performed by means of duplex scanning and physical examination. Duplex scanning is simple and cost-effective and, using duplex mapping, defines individual patient anatomy with considerable precision and provides valuable information that supplements the physician's clinical impression. The superiority of color flow duplex scanning over clinical examination for presurgical mapping has been well documented. Although ultrasound determination of reflux at the junctions and at specific locations above and below the knee may be adequate for diagnosis and epidemiological studies, preoperative mapping must include the entire length of the saphenous veins and the collateral veins. Such mapping can lead to selective surgical treatment and avoidance of complications related to extensive surgery.

Other new applications of noninvasive diagnostic methods that have completely changed our therapeutic approach are evaluation of indication and follow-up of venous stenting, acute deep venous obstruction, iliocaval localization, and chronic iliac obstruction, such as in May-Turner syndrome.

A new perspective is the study of chronic cerebrospinal venous insufficiency. Noninvasive evaluation of morphology and hemodynamic patterns in the intracranial and extracranial venous systems can provide interesting information about alterations in the cerebral venous system, but the sensitivity and specificity of the method and its accuracy are still not 
clearly defined. Further studies are needed, but the future in this regard is interesting.

In conclusion, the most important factor in determining a good treatment outcome is making an accurate diagnosis. ${ }^{2}$ Recognizing common clinical patterns of vascular disease is important, but with color flow duplex scanning now readily available to many providers, direct visualization and mapping of vascular pathways is possible for both acute and chronic diseases. ${ }^{3}$ This will ensure not only effective treatment of all abnormal vascular segments but also preservation of normal ones.

\section{References}

1. Antignani PL, Gossetti B, Righi D, et al. Vascular diagnostics laboratory. Workloads and indications. Minerva Cardioangiol. 2004;52:447-464.

2. Antignani PL, Gossetti B, Righi D, et al. Accreditation process for the performance of ultrasound vascular diagnostics procedures. Minerva Cardioangiol. 2005;53:485-508.

3. Antignani PL, Benedetti Valentini F, Aluigi L, et al. Guidelines for diagnosis of vascular diseases - ultrasound investigations - edited by Italian Society for Vascular Investigation. Int Angiol. 2012;31:1-77.

\section{Publish your work in this journal}

Journal of Vascular Diagnostics is an international, peer-reviewed journal of diagnostics, focusing on non invasive vascular investigation methods involved in the evaluation of vascular diseases. The journal is committed to the rapid publication in the fields of vascular diseases. Original research, review, case reports, expert opinion and commentaries

\section{Dovepress}

are all considered for publication. The manuscript management system is completely online and includes a very quick and fair peer-review system, which is all easy to use. Visit http://www.dovepress.com/testimonials.php to read real quotes from published authors. 